\title{
Model Construction of Material Distribution System Based on Digital Twin
}

\author{
Yunrui Wang ( $\nabla$ wangyunr2001@xust.edu.cn ) \\ Xi'an University of Science and Technology \\ Ziqiang Jiang \\ Xi'an University of Science and Technology \\ Wu Yue \\ Xi'an University of Science and Technology
}

\section{Research Article}

Keywords: Digital twin, full cycle material distribution, LSTM prediction, route optimization

Posted Date: November 1st, 2021

DOI: https://doi.org/10.21203/rs.3.rs-1018310/v1

License: (c) (1) This work is licensed under a Creative Commons Attribution 4.0 International License.

Read Full License 


\title{
Model construction of material distribution system based on
}

\section{digital twin}

\author{
Yunrui Wang ${ }^{1}$. Ziqiang Jiang ${ }^{1}$. Wu Yue ${ }^{1}$ \\ Corresponding author: Yunrui Wang E-mail: wangyunr2001@ xust.edu.cn \\ ${ }^{1}$ School of Mechanical Engineering, Xi'an University of Science and Technology, \\ Xi'an, China,710054 \\ Received: $\quad$ Accepted:
}

\begin{abstract}
Aiming at the problems of poor periodicity of workshop material distribution, difficult prediction of station material demand time node and redundant distribution route, this paper proposes a model construction method of material distribution system based on digital twin. Build a material distribution control mode based on digital twin, and establish a full cycle material distribution mechanism on this basis to comprehensively optimize the distribution cycle from the material preparation stage, dynamic replenishment stage and data transmission stage of adjacent distribution cycles. Build the digital twin model of material distribution system, establish the material demand time node prediction operation mechanism based on LSTM, accurately predict the station material demand time node, establish the material distribution route optimization model with the lowest total cost, and optimize the AGV route. Finally, it is applied to the asynchronous card line workshop of A enterprise to verify the effectiveness of this method.
\end{abstract}

Key words: Digital twin, full cycle material distribution, LSTM prediction, route optimization

\section{Introduction}

Material distribution in manufacturing industry refers to the supply and distribution of raw materials and parts by upstream suppliers to manufacturing enterprises, the distribution of raw materials at each station in the enterprise's production workshop, and the sales and distribution of products to customers by the company [1]. When the material distribution system (Material Distribution System, 
MDS) guides the actual material distribution in the workshop, the external of the workshop will be affected by the urgent order insertion of customers, and the internal of the workshop will be affected by personnel, equipment, materials, environment and other factors, such as equipment failure, personnel transfer, route blockage, etc., disturbing the material demand time node of the station. As a result, there is no orderly connection between distribution cycles, resulting in line material accumulation or shortage.

In order to solve the problem that it is difficult to determine the station material demand time node, Zhang Lianchao et al. proposed a material demand time prediction method based on grey theory to effectively reduce the error caused by worker differences [2]. Sheng Xia et al. proposed a method for predicting the completion time of rocket assembly based on trestle automatic encoder. This method can effectively improve the prediction accuracy by using the supervised learning process and parameter optimization process[3]. Niek tax et al. established a consistent model for predicting process monitoring tasks based on LSTM neural network, which proved that LSTM neural network has higher accuracy in time series prediction[4]. Xue Yan et al. Used LSTM deep neural network to model and predict Shanghai financial transaction data. Compared with BP neural network and traditional RNN neural network, it is proved that LSTM deep neural network can effectively predict stock market time series[5].

For the research of material distribution route, Chai, $\mathrm{H}$ et al. designed a two-stage multi-objective optimization algorithm for the whole container logistics distribution path planning of dangerous goods. This method can obtain the Pareto optimal iteration faster for the small-scale path problem[6]. Tang, s designed an improved hybrid algorithm based on Hopfield neural network and SA algorithm to improve the optimization and robustness of the algorithm. Sun[7]. YX et al. proposed a new adaptive genetic algorithm, which improves the local search ability through adaptive mutation, and can better solve the transportation path problem in distribution[8]. Jiang Zengqiang et al. designed a material distribution strategy based on dynamic cycle based on the distribution cost expectation model in uncertain 
environment, and established an optimization model aiming at the minimum distribution cost and maximum load factor, with an overall optimization range of 40\%[9]. Huang et al. emphasized the real-time data-driven logistics optimization method, and established a mathematical model with fuzzy time window based on the real-time manufacturing data collected by the Internet of things[10].

Reviewing the above-mentioned literature, certain research progress has been made in the prediction of the time node of the material demand at the station and the optimization of the material distribution path. However, the existing research does not have a clear phase division for the material distribution cycle, and the established prediction models are mostly static predictions, which cannot be dynamically predicted with the workshop production, which has certain limitations. When distributing materials, it only focuses on the distribution tasks in a single distribution cycle, ignoring the relevant production data generated in the completed distribution cycle, resulting in data waste and partial distortion of the distribution model, and it is impossible to realize the closed-loop control of workshop dynamic distribution.Therefore, new methods are urgently needed to establish a full-cycle material distribution mechanism, clarify the distribution tasks of each stage in the material distribution cycle, realize dynamic prediction and control during the replenishment process, and effective storage and transmission of workshop production data.

The emergence of digital twin technology provides an effective way to solve this problem. Digital twin is not a separate technology, it is a cross-fusion of multiple enabling technologies. It maps the data information in the physical entity to the operating environment of the twin model, and then analyzes, optimizes and revises the twin model, and then returns the data to the physical entity, thereby achieving a two-way relationship synchronous optimization between the physical entity and the twin model in terms of function and performance. Tao Fei put forward the construction criteria of digital twin model, established a set of digital twin model construction theoretical system, and provided theoretical support for the application and implementation of digital twin technology. Aiming at the coupling problems such 
as unreasonable production line layout and inaccurate logistics distribution[11], Guo het al. proposed a flexible cell manufacturing method based on digital twin, continuously simulated and optimized the twin model, and improved the line balance rate and per capita production capacity[12]. Wu et al. combined digital twin technology with planning and scheduling to implement control over uncertain factors[13]. To sum up, the introduction of digital twin technology can solve the problems of material demand time node disturbance, material route redundancy, and production data loss caused by the uncertainty of the workshop, and comprehensively optimize the material distribution cycle due to the characteristics of virtual and real integration and mutual driving.

Firstly, the paper integrates the digital twin technology with the material distribution system to form a closed-loop control mode of the material distribution system. On this basis, the full cycle material distribution mechanism is established, including the material preparation stage before production, the real-time dynamic replenishment stage in production and the data transmission stage of adjacent distribution cycles. In order to ensure the smooth operation of the control mode of the material distribution system and the orderly connection of each distribution cycle in the replenishment stage, build the digital twin model of the material distribution system, establish the material demand time node prediction model and material distribution route optimization model based on LSTM, and formulate the material distribution scheme with the lowest total distribution cost in line with the actual production of the workshop.

\section{Integration of digital twin and material distribution system}

\subsection{Establish control mode of material distribution system}

In order to ensure that the material distribution scheme accurately guides the actual material distribution process, the multi-scale material distribution system control mode shown in Figure 1 is established. 
The whole control mode is divided into three layers. The first is the real-time data acquisition of the physical layer. The sensor network composed of multiple sensors can real-time collect the production data generated by the material center, personnel, production line, AGV, RFID reader, etc. and the road environment data of the workshop, and transmit it to the information layer through the data interface.The second is the data-driven cycle of the information layer. The collected real-time data and workshop historical data are stored in the experience base of twin data, and the status and pose of production equipment are stored in the knowledge base. According to the collected real-time data, the geometric model, behavior model and rule model in the virtual workshop are updated to realize the real-time synchronous update and optimization of the virtual workshop and the physical layer. MDS determines the material demand node according to the simulation results and prediction results, formulates the material distribution scheme, feeds it back to the virtual workshop, and promotes the update of twin data. Thus, a data-driven cycle of virtual workshop, twin data and MDS is formed.The last is the encapsulation of system layer functions. It is mainly reflected in the accurate prediction of station material demand time node, the optimization of material distribution route and the real-time decision-making of distribution scheme.

In order to further divide the distribution tasks in the distribution cycle, optimize the distribution cycle and improve the distribution efficiency, a full cycle material distribution mechanism is established. The whole cycle material distribution mode is divided into three stages: material preparation before production, dynamic replenishment during production and data transfer in adjacent distribution cycles. The specific description is as follows:

(1) Material preparation stage before production.

The material preparation stage before production is also called the material laying stage. The first batch of materials required by the production station are delivered to the site according to the production plan[14]. The detailed process is shown in Figure 2.

After the daily production plan is released to the production management module 
of MES, MES invokes the relevant BOM to calculate the daily production plan according to the production data such as personnel, equipment and daily work hours in the current workshop. WMS formulates the material distribution plan according to the results of MES calculation, and prepares the first batch of materials meeting the station requirements, which are distributed to each station by AGV trolley. The real-time data generated by personnel, equipment, $\mathrm{AGV}$ and the first batch of materials in the material preparation stage will be collected as twin data and stored in the twin data platform.

(2) Real time dynamic replenishment stage in production

After the delivery of the first batch of materials, it is necessary to continuously feed materials to the site according to the material distribution scheme during the production process, which is called replenishment[15]. The whole feeding stage is shown in Figure 3.

Before production, the virtual workshop conducts preliminary simulation based on the highly realistic model with the physical workshop, combined with the material demand time node, road congestion and $\mathrm{AGV}$ usage predicted before production to obtain the preliminary material distribution scheme, and then optimizes the route in combination with the real-time data collected from the physical workshop, and simulates and iterates again, Then the optimized material distribution scheme is obtained. With the progress of the production process, the twin data platform checks the consistency between the real-time data collected in the physical workshop and the data simulated in the virtual workshop. If it passes the inspection, MDS will delegate the final generated material distribution scheme to WMS to obtain the replenishment plan and drive AGV to complete the material terminal distribution. On the contrary, the operation parameters of the virtual workshop are modified according to the real-time data of the physical workshop, and then the live simulation is carried out to generate the material distribution scheme in line with the actual situation, and the geometric model, behavior model and rule model of the virtual workshop are updated in real time to ensure the synchronization and high fidelity of the virtual workshop and the physical workshop. 
(3) Data transmission of adjacent distribution cycles

When an AGV completes the replenishment task of the Nth distribution cycle and returns to WMS, the distribution task of the current distribution cycle has been completed, but the life cycle of a series of twin data generated in the distribution activities of this distribution cycle does not end here. Instead, it is collected by sensors all over the workshop in real time and passed to the twin data platform through the data interface. After filtering and noise reduction processing, the useful data is passed to the $\mathrm{N}+1$ th distribution cycle to complete the data transfer. For example, the absence and leave of personnel in the Nth cycle, whether the health status of AGV is enough to support it to complete the next distribution, whether there are equipment faults, maintenance, shutdown and accumulation in the production line, the quantity of materials remaining at each station and the degree of road congestion, etc., all the above data can be used as the operation parameters of the $\mathrm{N}+1$ th cycle. The data transfer process is shown in Figure 4.

The twin data can be used as the carrier in the adjacent distribution cycles to realize the closed-loop transmission of material distribution data, solve the data consistency problem between the virtual workshop and the physical workshop, realize the virtual real interaction, generate the material distribution scheme in line with the actual production, and guide the actual material distribution work.

\subsection{Building a digital twin model of the material distribution system}

The smooth operation of the control mode of the material distribution system needs the support of the digital twin model of the material distribution system. The whole twin model is built around the physical workshop. The twin model is mainly composed of five modules: physical workshop, virtual workshop, twin data platform, data prediction and MDS. The twin data platform module mainly includes the real-time data of the physical workshop, the operation data of the virtual workshop and the data consistency test. The data prediction module mainly refers to the prediction of the station demand time node. The specific composition of the digital twin model of the material distribution system is shown in Figure 5. 


\subsubsection{Physics workshop}

The physical workshop is the sum of all physical entities, including personnel, production lines, materials, AGV, road environment, etc[16]. The physical workshop is the main carrier of all production activities and the cornerstone of the operation of the digital twin model of the entire material distribution system. The workshop is mainly divided into three levels, namely the unit level, the system level and the complex system level.Unit layer refers to the smallest unit that can complete production functions, including production station, AGV, etc; The system layer refers to the level that can complete production tasks composed of various equipment. It is the addition of production factors such as material warehouse, line side warehouse, road environment and production line on the basis of the unit layer; The complex system layer refers to the comprehensive control of the whole material distribution process, the collection and recording of all aspects of material flow and information flow, and the real-time interaction and feedback with the virtual space to ensure the continuous and effective operation of production.

\subsubsection{Virtual workshop}

Virtual workshop is a digital workshop that uses three-dimensional simulation modeling software (such as AutoCAD, SketchUp, 3ds max, Solidworks, etc.) to describe the physical workshop in multi-dimensional detail on the computer[17]. It can interact and feed back with the physical workshop in real time. It is an important part of the complete operation of the whole twin model. Virtual workshop is the digital image of the physical Workshop on the computer. It is also composed of unit layer, system layer and complex system layer. The unit layer of the workshop mainly loads the distribution tool model, including AGV trolley model, etc; After loading the basic unit layer model, add relevant distribution constraints (such as the shortest total distribution time, the shortest travel distance of AGV trolley, the smallest distribution cost, etc.) to form a material distribution model to form a complete workshop system layer; On this basis, coordinate and manage the information flow collected from the physical workshop and the material flow of the storage system to form a complex system layer. 


\subsubsection{Twin Data Platform}

Twin data platform is a comprehensive management platform that stores the physical data of the physical workshop, the digital information of the virtual workshop and the derived data generated in the process of their interaction[18]. It can not only collect and transfer the data, but also check the data consistency internally, so as to support the material distribution system to make correct decisions. The physical data of the physical workshop mainly includes the number of stations, geometric parameters (such as shape, size, position, etc.) and status of production equipment. It can be collected through the sensor network composed of sensors all over the physical workshop, and then transmitted to the twin data platform through the data interface. The digital information of the virtual workshop mainly includes the loaded equipment model, material distribution model, etc., as well as the data generated by the simulation, verification, evaluation and other activities based on the loaded model, and a series of data generated by the interaction and mapping between the two workshops at all levels. Through the integration of multi-level, multi-temporal and spatial correlation data and historical information, an information physical big data platform is formed to break the information islands in the physical workshop and virtual workshop, and use the twin data as the link to drive the continuous and efficient operation of virtual workshop simulation activities, so as to connect the whole material distribution system and realize the sharing, interworking and closed-loop flow of workshop information.

\subsubsection{Data prediction}

The data prediction module can directly call the relevant data of the twin data platform, and then predict the uncertain factors of the workshop in combination with the corresponding prediction model, such as equipment availability prediction, production plan change prediction, station material demand time node prediction, etc. The prediction of station material demand time node refers to the prediction from the perspective of workers' processing history data, workers' proficiency and workers' fatigue. Its purpose is to predict the accurate material demand time node and provide the basis for the formulation of material distribution scheme. 


\subsubsection{MDS}

The core function of MDS is to formulate a real-time material distribution scheme in line with the actual production of the workshop according to the accurate data provided by the twin data platform. In the material preparation stage before the start of material distribution activities, a series of preparation activities need to be carried out, such as material counting, BOM decomposition, distribution tool preparation, etc. With the continuous progress of the production process and the continuous interaction between the virtual and real workshops, the data analysis and noise reduction processing are carried out for the prediction results of the material demand time node, and then the material distribution path optimization model is loaded to generate the material distribution scheme and complete the dynamic replenishment stage. The real-time data generated in the distribution cycle is stored in the twin data platform to facilitate the next distribution cycle.

\section{Material demand time node forecast based on LSTM}

The dynamic replenishment stage in the whole cycle material distribution is inseparable from the accurate prediction of the material demand time node, and the key factor determining the material demand time node is the material consumption time of the station. The material consumption time of the station is affected by the operation proficiency, fatigue and station characteristics of workers. Therefore, the accurate prediction of the material consumption time of the station is the basis for the prediction of the material demand time node. The prediction of material consumption time belongs to a time series problem. Here, a material demand time node prediction method based on LSTM (long short term memory) is proposed, and its operation mechanism is shown in Figure 6.

After the production starts, the stations participating in the production are the stations that need to be predicted. The historical material consumption time, worker proficiency, fatigue and other data of the required predicted stations are obtained from the twin data platform. On this basis, the modeling rules and constraints are called from the knowledge base to establish the LSTM prediction model for each station. After initialization, optimization and training, the model loads relevant data to predict 
material consumption time, demand time node and demand time window, eliminates abnormal values, generates prediction value sequence and transmits it to MDS. MDS calls AGV usage, road environment and other data from the experience base of twin data platform to formulate material distribution scheme to guide actual material distribution, and the obtained distribution information is fed back to the experience base of twin data platform for real-time update. If the AGV terminal distribution is inconsistent with the station material demand time window when MDS guides the actual material distribution, feed back the actual material demand time window to the dynamic prediction module, adjust the LSTM prediction model and update the knowledge base in real time.

\subsection{Establish an LSTM neural network model}

The long-term and short-term memory network (LSTM) is a variant of the recurrent neural network (RNN). Therefore, the two have certain similarities in structure, both of which contain the external circulation structure, while the LSTM also has the internal circulation structure of memory cells[19]. The hidden layer of LSTM includes forgetting gate, input gate, output gate and cell state, which can ensure that the signal will not explode or disappear after multiple cycles, so that the information can be transmitted completely. Its network structure is shown in Figure 7. Where $c_{t-1}$ and $c_{t}$ are the cell state of the previous time and the cell state of the current time respectively, $y_{t-1}$ and $y_{t}$ are the output of the previous time and the output of the current time respectively, and $x_{t}$ is the input of the current time, $\sigma$ is the sigmoid activation function, tanh is the tanh activation function, $f_{t}, i_{t}$ and $o_{t}$ are the number of sequences between $[0,1]$ generated by the sigmoid activation function, and $c^{*}{ }_{t}$ is the new cell state generated by the tanh activation function.

\subsection{Material demand time node forecast}

In the established LSTM neural network model, the worker's historical material consumption time, daily working hours, fatigue and other related parameters can be input to establish the worker's independent prediction model. The output result of the model is the time when workers consume a single material, and the material demand time node is the time point when workers consume materials to the safety stock limit. 
The time period from this time point to the complete consumption of materials is the station material demand time window, as shown in Figure 8.

AGV needs to respond as quickly as possible to all stations that issue material requirements within the material demand time window $\left[\mathrm{a}_{\mathrm{i}}, \mathrm{b}_{\mathrm{i}}\right]$, so it is necessary to optimize the material distribution route of $\mathrm{AGV}$ to ensure that all stations that issue material requirements can be met within the demand time window under the condition of the lowest total distribution cost, so as to improve the efficiency of material distribution.

\section{Establish the optimization model of material distribution route with the lowest}

\section{total cost}

The essence of the material distribution routing problem is the vehicle routing problem with soft time window (VRPTW). The arrival of distribution vehicles earlier than the time window will lead to material accumulation, and the arrival later than the time window will lead to production line interruption. In order to ensure that there is no biological material accumulation and interruption in the workshop production line, the distribution vehicles should arrive within the specified time window.

\subsection{Establish a soft time window}

The soft time window means that the delivery vehicle is allowed to arrive within a certain period of time before or after the station demand time window. When the delivery vehicle arrives outside the specified time window, a corresponding penalty fee will be incurred[20]. The penalty fee changes with time. The function can be set as follows:

$$
H(t) \begin{cases}T_{e} * a_{i}^{e} & \left(t_{i}<a_{i}\right) \\ 0 & \left(a_{i}<t_{i}<b_{i}\right) \\ T_{l} * b_{i}^{e} & \left(t_{i}>b_{i}\right)\end{cases}
$$

Function Description: $H(t)$ is the time penalty function, $t_{i}$ represents the time when the logistics vehicle arrives at the specified station $i, a_{i}$ represents the earliest arrival time of the station $i$ time window, $b_{i}$ represents the latest arrival time of the station $i$ time window, $T_{e}$ is the penalty when the distribution vehicle arrives at the 
station ahead of the specified time window, and $T_{l}$ is the penalty when the distribution vehicle arrives behind the specified time window, $a_{i}^{e}$ and $b_{i}^{e}$ are negatively and positively correlated with time, respectively. When the logistics vehicle arrives within the specified time window, the penalty number is 0 . When the logistics vehicle arrives earlier than the specified time window, the penalty number is $T_{e} * a_{i}^{e}$, when the logistics vehicle arrives later than the specified time window, the penalty number is $T_{l} * b_{i}^{e}$.

\subsection{Establishment of multi-target VRPTW model}

Set the relevant parameters of the established multi-objective VRPTW model: the production workshop has 1 distribution center and $N$ vehicles for distribution activities. The maximum capacity of each vehicle is the same as $M$, and $K$ stations need to be distributed. Station 0 represents the distribution center, the demand of station $i$ is $q_{i}$, and the starting and ending point of each distribution vehicle can only be the distribution center, each station must be distributed and can only be distributed by one vehicle. The demand time window of station $i$ is $\left[a_{i}, b_{i}\right]$. The specific model is as follows:

$$
\begin{array}{cc}
\min A=\sum_{n=1}^{N} \sum_{i=1}^{K}\left\{\max \left[\left(a_{i}-t_{i n}\right), 0\right]+\max \left[\left(t_{i n}-b_{i}\right), 0\right]\right\} & \min B=\sum_{i=0}^{K} \sum_{j=0}^{K} \sum_{n=1}^{N} d_{i j} x_{i j n} \\
\sum_{j=1}^{K} x_{0 j n}=\sum_{j=1}^{K} x_{j 0 n} \quad \forall n \in\left[\begin{array}{ll}
1, & N
\end{array}\right] \\
\sum_{i=0}^{K} \sum_{j=0}^{K} q_{j} x_{i j n} \leq M & \forall n \in\left[\begin{array}{ll}
1, & N
\end{array}\right] \\
\sum_{i=0}^{K} \sum_{j=0}^{K} x_{i j n} \leq K & \forall n \in\left[\begin{array}{ll}
1, & N
\end{array}\right] \\
\sum_{n=1}^{N} \sum_{i=1}^{K} y_{n i}=K & (\mathrm{i}=1,2, \ldots . . k) \\
\sum_{n=1}^{N} y_{n i}=1 &
\end{array}
$$




$$
\begin{gathered}
\left\{\begin{array}{r}
a_{i}^{e}=\max \left(a_{i}-t_{i}, 0\right) \\
b_{i}^{e}=\max \left(t_{i}-b_{i}, 0\right)
\end{array}\right. \\
x_{i j n}\left\{\begin{array}{l}
1 \\
0
\end{array}\right. \\
y_{n i}\left\{\begin{array}{l}
1 \\
0
\end{array}\right.
\end{gathered}
$$

In the formula:

$N$ - Total number of distribution vehicles;

$M-$ Maximum capacity of distribution vehicles;

$T$ _ A positive integer with a large value;

$q_{j} \longrightarrow$ Demand of station $j$

$d_{i j} \_$Distance from station $i$ to station $j$;

$a_{i}$ - The earliest time when the delivery vehicle arrives at station $i$;

$b_{i}-$ The latest arrival time of delivery vehicle at station $i$;

$a_{i}^{e}{ }_{i}$ Time to arrive at station $i$ in advance;

$b_{i}^{e}$ - Time lag to station $i$

$t_{i j}$-Travel time from station $i$ to station $j$;

$t_{i n}$ The time when the delivery vehicle $n$ arrives at station $i$;

$y_{n i}$ - Identification function of whether station $i$ is distributed by vehicle $n$;

$x_{0 j n}-$ Distribution vehicle $\mathrm{n}$ from distribution center to station $j$;

$x_{j 0 n}$ - The distribution vehicle $n$ returns to the distribution center from station $j$;

$x_{i j n}$ When the value of $x_{i j n}$ is 1 , it means that the distribution vehicle $n$ travels to station $j$ after distribution station $i$, and the value of $x_{i j n}$ in other cases is 0 .

Formula (2) indicates that the difference between the delivery vehicle and the time window is the smallest when it arrives at the corresponding station; Formula (3) indicates that the total distance traveled by the distribution vehicle when performing the distribution task is the shortest; Formula (4) indicates that all distribution vehicles start from the distribution center and return to the distribution center after performing the distribution task; Formula (5) indicates that the materials loaded by all distribution 
vehicles cannot exceed the maximum capacity; Formula (6) indicates that the number of distribution stations does not exceed the total number of stations; Formula (7) indicates that each station should be distributed; Formula (8) indicates that each station can only be distributed by one vehicle; Formula (9) indicates that the delivery vehicle arrives earlier than the specified time window or later than the specified time window, and can only be one of them.

\subsection{Processing of multi-target VRPTW model}

The two objectives in the VRPTW model can be expressed by the unified dimension of cost, so the linear addition in the unified objective method is used to transform the multi-objective function. The process of applying linear addition to transform multi-objective VRPTW model is as follows:

Step 1: Analyze specific problems and get the multi-objective function to be optimized. The multi-objective functions to be transformed in VRPTW model are formulas (2) and (3):

Step 2: Analyze multi-objective functions, find out their commonalities, and give certain coefficients to convert them into dimensional unified functions. In this VRPTW model, through the analysis of the current situation of logistics distribution, it can be seen that all the costs generated in distribution activities can be included by the total distribution cost. For the objective function $A$, the penalty coefficient $e$ is given when the vehicle arrives at the station in advance, and the penalty coefficient $l$ is given when the vehicle lags behind; For the objective function $B$, it can be transformed into the vehicle driving cost function in combination with the cost $f_{n}$ required by the vehicle driving unit distance. In addition, the fixed cost $C$ of vehicle start-up should be added. The transformed multi-objective function is as follows:

$$
\begin{gathered}
\min A^{\prime}=\sum_{n=1}^{N} \sum_{i=1}^{K}\left\{e * \max \left[\left(a_{i}-t_{i n}\right), 0\right]+l * \max \left[\left(t_{i n}-b_{i}\right), 0\right]\right\} \\
\min B^{\prime}=\sum_{i=0}^{K} \sum_{j=0}^{K} \sum_{n=1}^{N} f_{n} * d_{i j} x_{i j n} \\
C=p^{* h}
\end{gathered}
$$

In the formula: 
$e$ - Penalty coefficient per unit time for delivery vehicles arriving earlier than the specified time window;

$l$ - Penalty coefficient per unit time for delivery vehicles arriving later than the specified time window;

$h-$ Number of vehicles started;

$p-$ Start up cost of a single distribution vehicle;

$f_{n}$ - Unit distance cost of vehicle $n$.

Step 3: Convert the multi-objective function with unified dimensions into a single objective function. In this VRPTW model, the total distribution cost function $F$ can be introduced to convert the multi-objective function with the shortest vehicle travel distance and the smallest time window difference into a single objective function with cost as a single dimension. To sum up, the objective function of VRPTW model is:

$$
F=A^{\prime}+B^{\prime}+C
$$

\section{Case}

Based on the above research, this paper carries out an example application to the asynchronous card line workshop of enterprise A. Asynchronous line is a production line for down procedure and testing and packaging of asynchronous cards. The workshop size is $16 \mathrm{~m} \times 11 \mathrm{~m}$, there are 6 down procedure and test stations, 3 packaging stations and 9 stations in total in the workshop. There are $4 \mathrm{AGV}$ in the workshop, numbered AGV01, AGV02, AGV03 and AGV04. The traveling speed of AGV trolley is in the range of $0.25-0.45 \mathrm{~m} / \mathrm{s}$, and the speed is stable and controllable. The workshop layout is shown in Figure 9, where each grid is $0.5 \mathrm{~m} \times 0.5 \mathrm{~m}$ 。

The asynchronous card line produces various types of asynchronous cards for different asynchronous control systems. The material BOM of each asynchronous card is not exactly the same, and when the asynchronous card type produced on the production line is switched, the production process is adjusted accordingly, and the material demand quantity, material demand time node and AGV driving route of relevant stations are also changed. Therefore, the asynchronous card MSD300, which accounts for the largest proportion of monthly output, is applied as an example. 


\subsection{Asynchronous card line workshop material distribution operation mechanism based on digital twins}

Establish a material distribution operation mechanism based on digital twin for asynchronous card line workshop, as shown in Figure 10.

The operation mechanism of material distribution in asynchronous card line workshop based on digital twin can realize the real-time control of material distribution process. The real-time data collected from the asynchronous line workshop through the sensor network is stored in the experience base of the twin data platform. The LSTM dynamic prediction module calls the LSTM prediction model in the knowledge base for prediction, and the obtained results are transmitted upward to the virtual space and downward to the twin data platform. The virtual workshop forms a preliminary material distribution scheme through two-way interaction with the twin data platform, and continuously simulates and optimizes the optimized material distribution scheme, which is used as the basis to clearly divide the tasks of each stage of the whole cycle material distribution. If the data consistency check is passed, the asynchronous line workshop will guide the actual distribution according to the material distribution scheme generated in the virtual workshop. If it fails, it will return to the twin data platform to modify the relevant parameters of the virtual workshop according to the real-time data of the asynchronous line workshop for live simulation.

\subsection{LSTM-based material demand time node prediction}

Take the down procedure and test station 1 as an example, retrieve the historical data of station 1, and establish the LSTM material demand time node prediction model for prediction. In the model, the sequence length is set as 50 , the ratio of training set to test set is 9:1, the actual operation time of station 1 is shown in Table 1, and the prediction results are shown in Table 2.

The LSTM based material demand time node prediction method is compared with the ordinary moving average method and Holt linear trend method. The prediction results are shown in Figure 11 and the error comparison is shown in Figure 12 The general moving average method means that the historical data of station 1 are translated and averaged item by item to predict; Holt linear trend method refers to 
adding trend prediction equation on the basis of station 1 historical data.

As can be seen from Fig. 11 and Fig. 12, the prediction method based on LSTM proposed in this paper is closer to the actual operation time than the ordinary moving average method and Holt linear trend method in the prediction time scale, with higher prediction accuracy and time series ductility, and the prediction average error rate is $4.01 \%$, which can provide more accurate data prediction support for material distribution and ensure the normal production of the workshop.

\subsection{Establish a material distribution route optimization model}

\subsubsection{Establish material demand time window}

Establish LSTM forecasting models for 9 stations of asynchronous card line to forecast material demand time window. According to the relationship between the material consumption time and the demand time window, the material demand time window of each station is calculated and sorted as shown in Table 3:

According to the predicted station demand time window, station 2 sends the material distribution demand first, the time point of station 2 sending the material demand is $31 \mathrm{~min}$, and the latest delivery time is $53 \mathrm{~min}$. Within this demand time window, stations $1,3,4,5,6,7,8$ and 9 send the material distribution request at the same time. In this distribution cycle, the 9 stations that send material requests are reordered by $1-10$, and the distribution center is represented by station 0 . The location coordinates, distribution quantity, demand time window, loading and unloading time of the 9 stations are known, as shown in Table 4:

\subsubsection{Determination and solution of VRPTW model parameters of asynchronous} card line

According to the actual situation of asynchronous line, the parameters in VRPTW model are set reasonably. The specific settings are shown in Table 5:

After the model is established, relevant software is used to solve it. The obtained AGV distribution route is shown in Figure 13. In the figure, the geometric center of each station is taken as the AGV distribution end point, so the obtained distribution route is the approximate route of $\mathrm{AGV}$. 
Sort out the results and AGV distribution route to obtain the complete route and total cost of this material distribution, as shown in Table 6 , in which the unit of each cost is yuan.

Compare the material distribution schemes before and after optimization, and the results are shown in Table 7.

For asynchronous card line workshop, the average prediction error rate of LSTM prediction model is about $4.01 \%$. Based on the analysis of material distribution route, the results show that compared with before optimization, the number of vehicle starts is reduced by $40 \%$, the vehicle full load rate is increased by $36.68 \%$, the total distribution cost is reduced by $24.45 \%$, and all stations are distributed.

\section{Conclusion}

This paper studies the application of digital twin technology in the process of material distribution, and constructs the control mode of material distribution system based on digital twin and the digital twin model of material distribution system. On this basis, a three-stage full cycle material distribution mechanism based on material preparation stage, dynamic replenishment stage and adjacent distribution cycle data transmission is proposed to comprehensively optimize the material distribution cycle. In the material preparation stage, MES and WMS are connected to realize the distribution of the first batch of materials at the station; In the dynamic replenishment stage, the material demand time node prediction operation mechanism based on LSTM and the material distribution route optimization model with the lowest total cost are established to realize the accurate prediction of station material demand time node and the optimization of AGV distribution route; In the data transmission stage, based on the twin data platform, the production data generated in the distribution cycle are collected in real time, processed and stored effectively, and transmitted to the next distribution cycle. Based on the real-time data, the continuous process of material distribution is promoted. Through the example of asynchronous card line workshop, the effectiveness of the proposed method is verified, which provides a practical basis for realizing the material distribution system based on digital twin. However, the full cycle material distribution mechanism based on digital twin 
technology needs to be further subdivided. In the future, further research will be made on the dynamic replenishment stage in order to improve the distribution mechanism and promote the implementation and application of digital twin technology.

\section{Declarations:}

\section{Ethical Approval}

All authors declare that this article does not have any academic ethics issues and strictly follows the journal submission rules

\section{Consent to Participate}

All authors agree to participate in the research work of this paper and publish it in the International Journal of Advanced Manufacturing Technology

\section{Consent to Publish}

All authors agree to publish this article in the International Journal of Advanced Manufacturing Technology

\section{Funding information}

This work was supported by the [General program of National Natural Science Foundation of China] under Grant [number 52074210].

\section{Conflict of interest}

The author(s) declare no potential conflicts of interest with respect to the research, authorship and/or publication of this article.

\section{Acknowledgements}

The authors wish to acknowledge support from Staff of Industrial Engineering Project Team, School of Mechanical Engineering, Xi'an University of Science and Technology

\section{Authors' contributions}

All authors contributed equally to the generation and analysis of experimental data, and the development of the manuscript.

\section{Reference}

[1]Zheng, Y, Qiu, S, Shen, F, \& He, C. (2020). RFID-based material delivery method for mixed-model automobile assembly. Computers \& Industrial Engineering, 139, 
106023.https://doi.org/10.1016/j.cie.2019.106023.

[2]Zhang Lianchao, Liu Weiran, Cheng Jiangfeng, Tao Fei, Meng Shaohua \&amp; Chen Changyu. (2020). Just in time material distribution method in digital twin workshop of satellite assembly. Computer integrated manufacturing system (11), 2897-2914. doi: 10.13196/j.cims.2020.11.001.

[3] Sheng Xia, Zhao Xinming, Zhang Peng, Zhang Jie, Cheng Hui, Liu Siqi \&amp; Zhang Chunyu. (2019). Prediction of completion time of rocket assembly based on trestle automatic encoder. Computer integrated manufacturing system (11), 2720-2730. doi: 10.13196/j.cims.2019.11.003.

[4] Tax, N, Verenich, I, La Rosa, M, \& Dumas, M. (2017, June). Predictive business process monitoring with LSTM neural networks. In International Conference on Advanced Information Systems Engineering (pp. 477-492). Springer, Cham.

https://doi.org/10.1007/978-3-319-59536-8_30.

[5] Yan, X, Weihan, W, \& Chang, M. (2021). Research on financial assets transaction prediction model based on LSTM neural network. Neural Computing and Applications, 33(1), 257-270.https://doi.org/10.1007/s00521-020-04992-7.

[6]Chai, H, He, R., Ma, C., Dai, C., \& Zhou, K. (2017). Path planning and vehicle scheduling optimization for logistic distribution of hazardous materials in full container load. Discrete Dynamics in Nature and Society, 2017.

https://doi.org/10.1155/2017/9685125.

[7] Tang, S, Zhao, H, Wang, Z, Zhu, Y, Hou, D, \& Wang, H. (2021). Analysis of the material distribution system of wise information technology of 120 under deep learning. The Journal of Supercomputing, 1-15.

https://doi.org/10.1007/s11227-021-03646-2.

[8]Sun, Y, Geng, N, Gong, S, \& Yang, Y. (2020). Research on improved genetic algorithm in path optimization of aviation logistics distribution center. Journal of Intelligent \& Fuzzy Systems, 38(1), 29-37.https://doi.org/10.3233/JIFS-179377.

[9]Jiang Zengqiang, Jin Yang, Liang Junyi \&amp; E Mingcheng. (2017). Dynamic material distribution strategy of mixed model assembly workshop under uncertain environment. Computer integrated manufacturing system (10), 2108-2118. doi: 
10.13196/j.cims.2017.10.004.

[10]Huang, S, Guo, Y, Zha, S, \& Wang, Y. (2019). An internet-of-things-based production logistics optimisation method for discrete manufacturing. International Journal of Computer Integrated Manufacturing, 32(1), 13-26.

https://doi.org/10.1080/0951192X.2018.1550671.

[11]Tao Fei, Zhang He, Qi Qinglin, Xu Jun, sun Zheng, Hu Tianliang, Liu Xiaojun, Liu Tingyu, Guan Juntao, Chen Changyu, Meng fanwei, Zhang Chenyuan, Li Zhiyuan, Wei Yongli, Zhu Minghao, Xiao bin(2021). Theory and application of digital twin model construction. Computer integrated manufacturing system (01), 1-15. doi: 10.13196/j.cims.2021.01.001.

[12]Guo, H, Chen, M, Mohamed, K, Qu, T, Wang, S, \& Li, J. (2021). A digital twin-based flexible cellular manufacturing for optimization of air conditioner line. Journal of Manufacturing Systems, 58, 65-78.

https://doi.org/10.1016/j.jmsy.2020.07.012.

[13]Wang YR, Wu ZL (2020) Model construction of planning and scheduling system based on digital twin. The International Journal of Advanced Manufacturing Technology (7-8):2189-2203. https://doi:10.1007/s00170-020-05779-9.

[14]Rahman, H. F, \& Nielsen, I. (2019). Scheduling automated transport vehicles for material distribution systems. Applied Soft Computing, 82, 105552. https://doi.org/10.1016/j.asoc.2019.105552.

[15]Li, L, Chen, Y, Lai, D, Li, B, \& Wei, X. (2020). Material distribution method in discrete manufacturing systems and a case study from engine builders. Proceedings of the Institution of Mechanical Engineers, Part B: Journal of Engineering Manufacture, 234(14), 1720-1728.https://doi.org/10.1177/0954405420933093.

[16]Jones, D, Snider, C, Nassehi, A, Yon, J, \& Hicks, B. (2020). Characterising the Digital Twin: A systematic literature review. CIRP Journal of Manufacturing Science and Technology, 29, 36-52.https://doi.org/10.1016/j.cirpj.2020.02.002.

[17]Liu, M, Fang, S, Dong, H, \& Xu, C. (2021). Review of digital twin about concepts, technologies, and industrial applications. Journal of Manufacturing Systems, 58, 346-361.https://doi.org/10.1016/j.jmsy.2020.06.017 
[18]Qi, Q, Tao, F, Hu, T., Anwer, N., Liu, A., Wei, Y., ... \& Nee, A. Y. C. (2019). Enabling technologies and tools for digital twin. Journal of Manufacturing Systems. [19]Sherstinsky, A. (2020). Fundamentals of recurrent neural network (RNN) and long short-term memory (LSTM) network. Physica D: Nonlinear Phenomena, 404, 132306.https://doi.org/10.1016/j.physd.2019.132306.

[20]Oyola J. The capacitated vehicle routing problem with soft time windows and stochastic travel times[J]. Revista Facultad de Ingeniería, 2019, 28(50): 19-33. https://doi.org/10.19053/01211129.v28.n50.2019.8782 
Figures
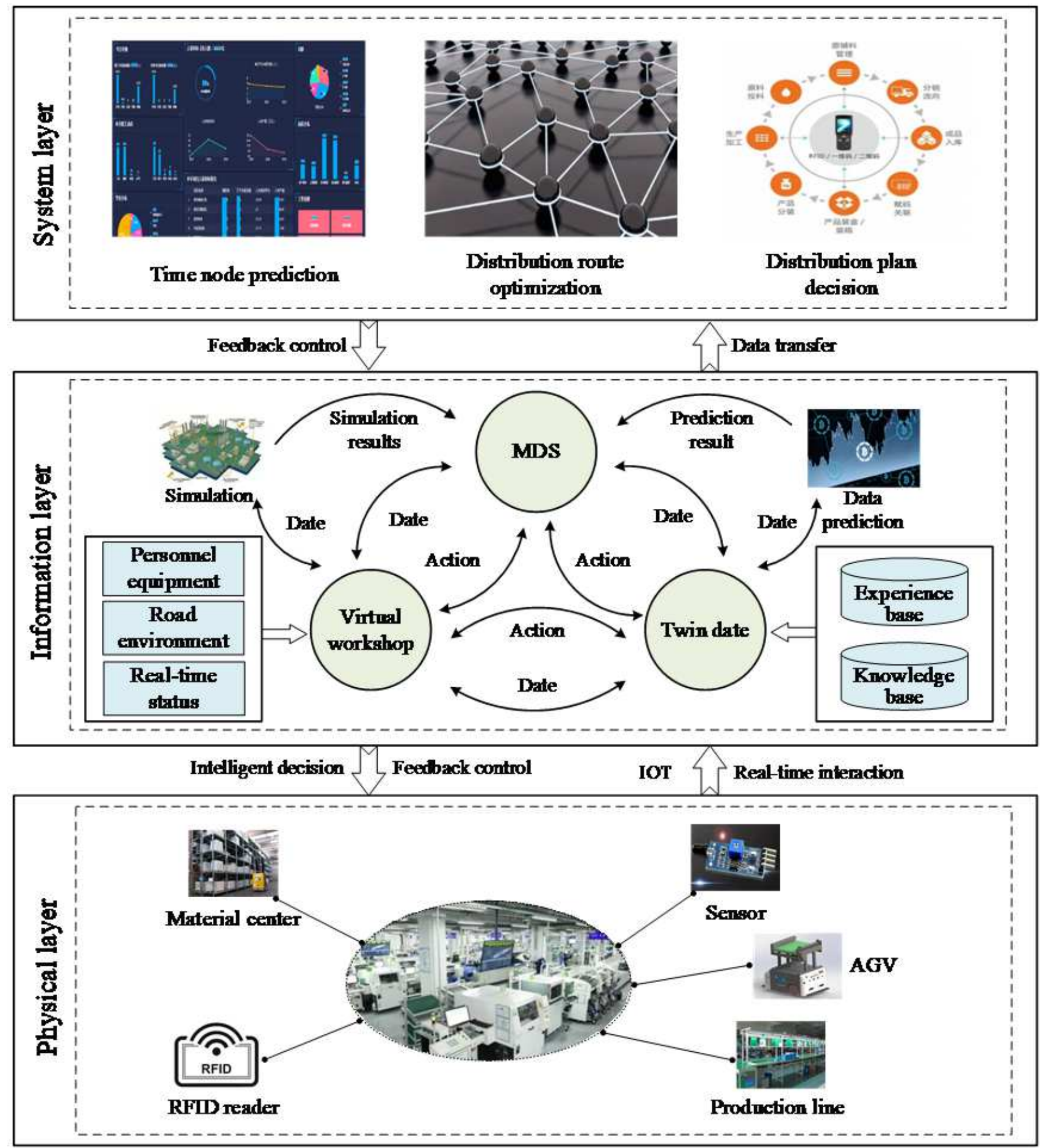

Figure 1

Control mode of material distribution system based on digital twin 


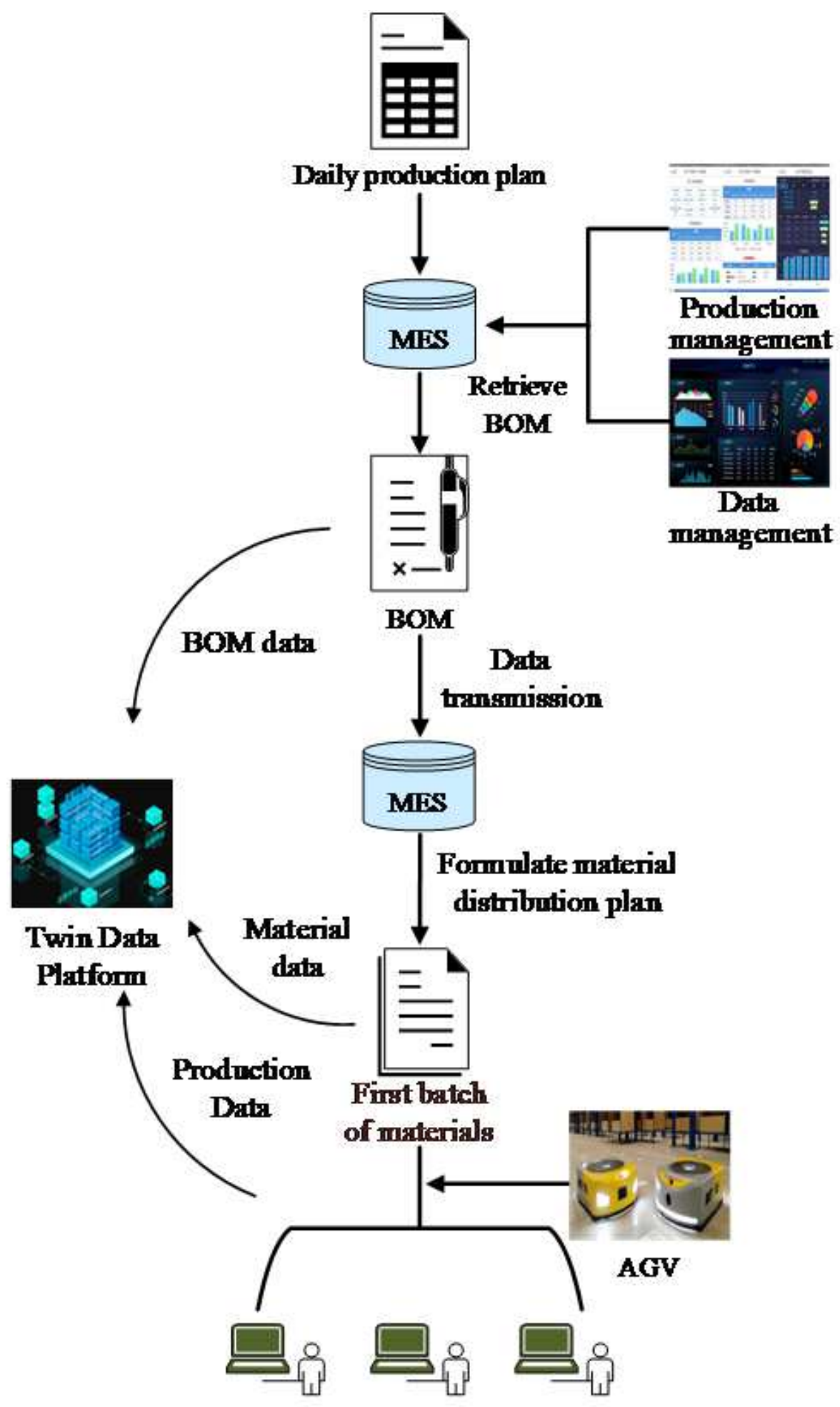

Figure 2

Material preparation stage 


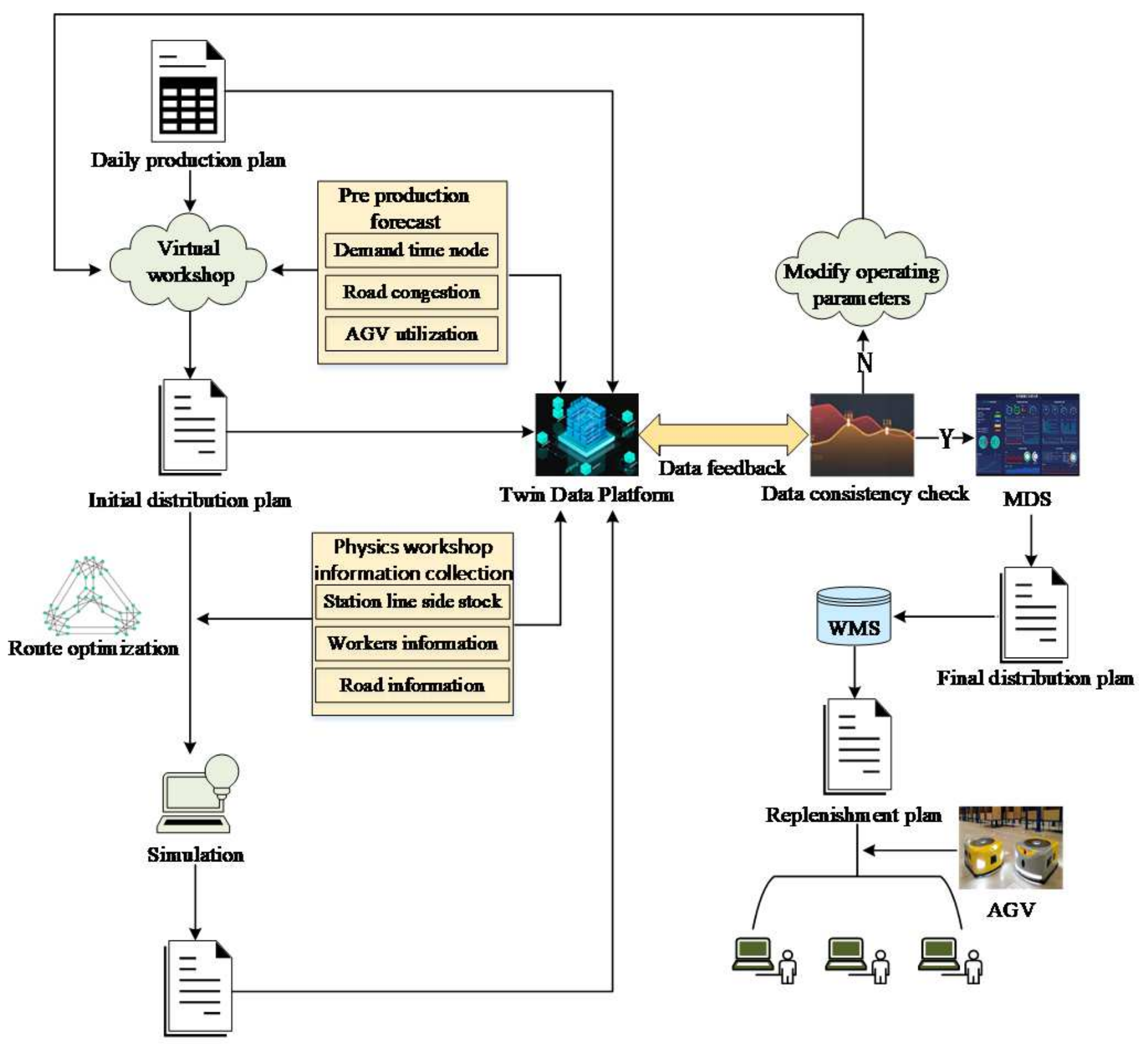

Optimized distribution plan

\section{Figure 3}

Dynamic feeding stage

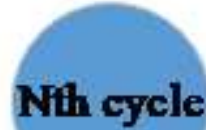

Data transfer
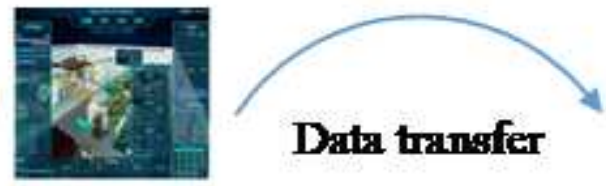

Twin system

(Data process)

Figure 4 


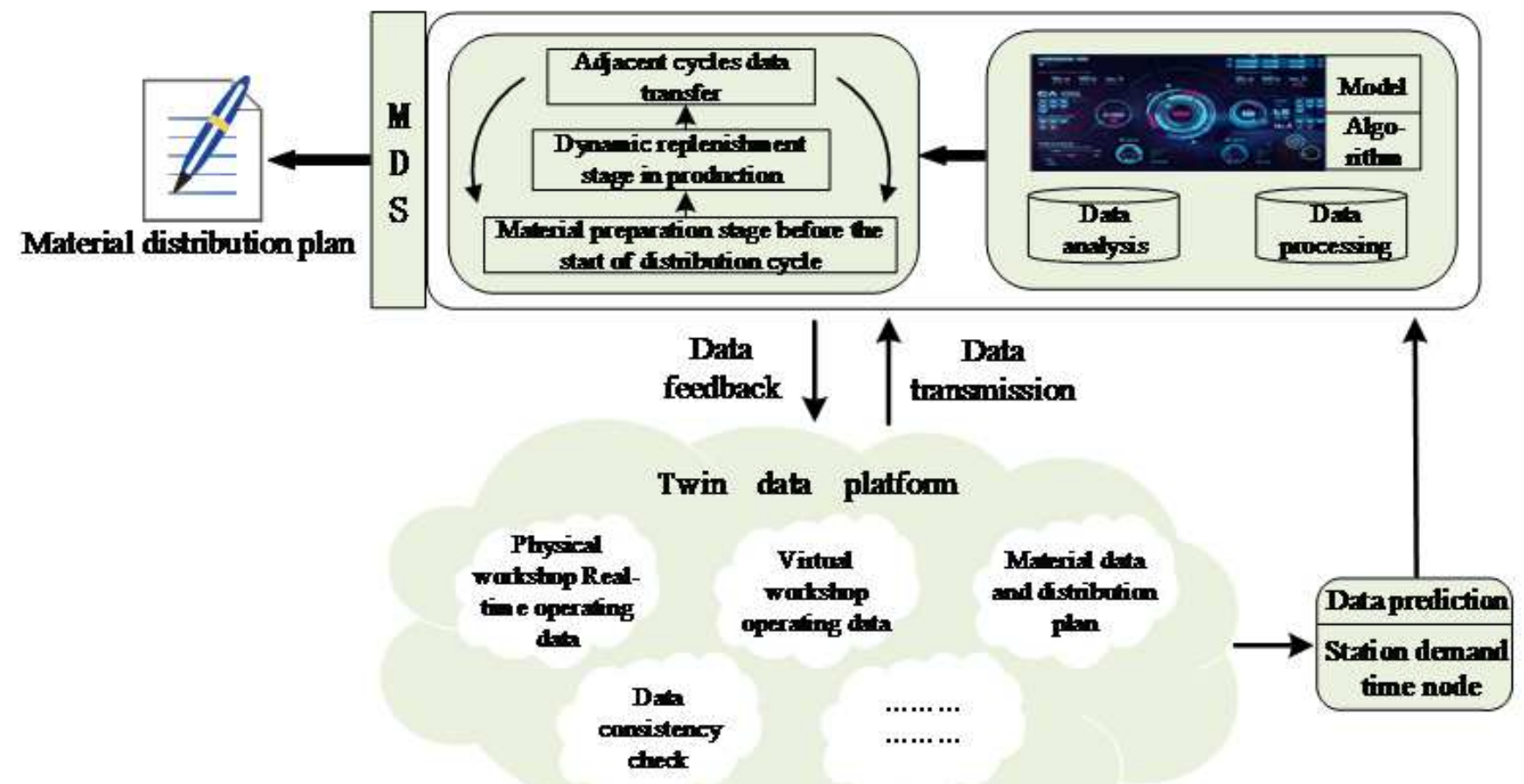

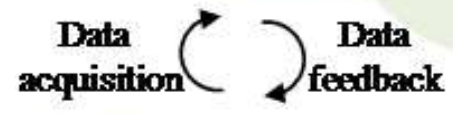

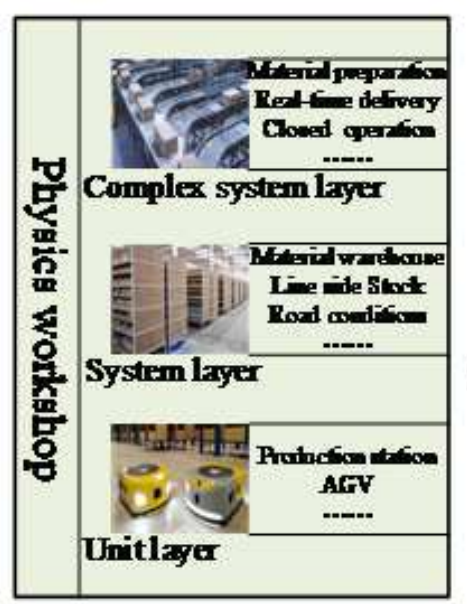

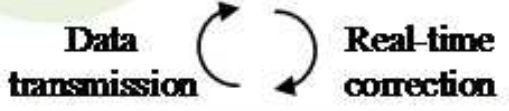

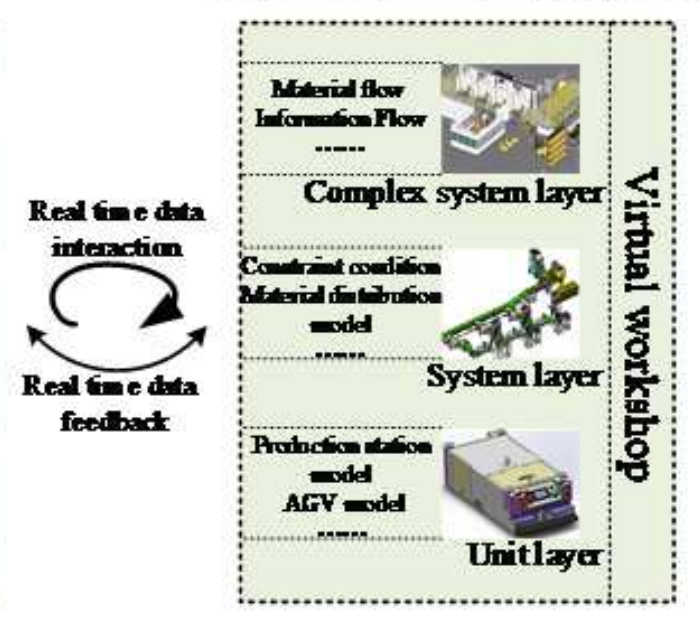

Figure 5

Digital twin model of material distribution system 


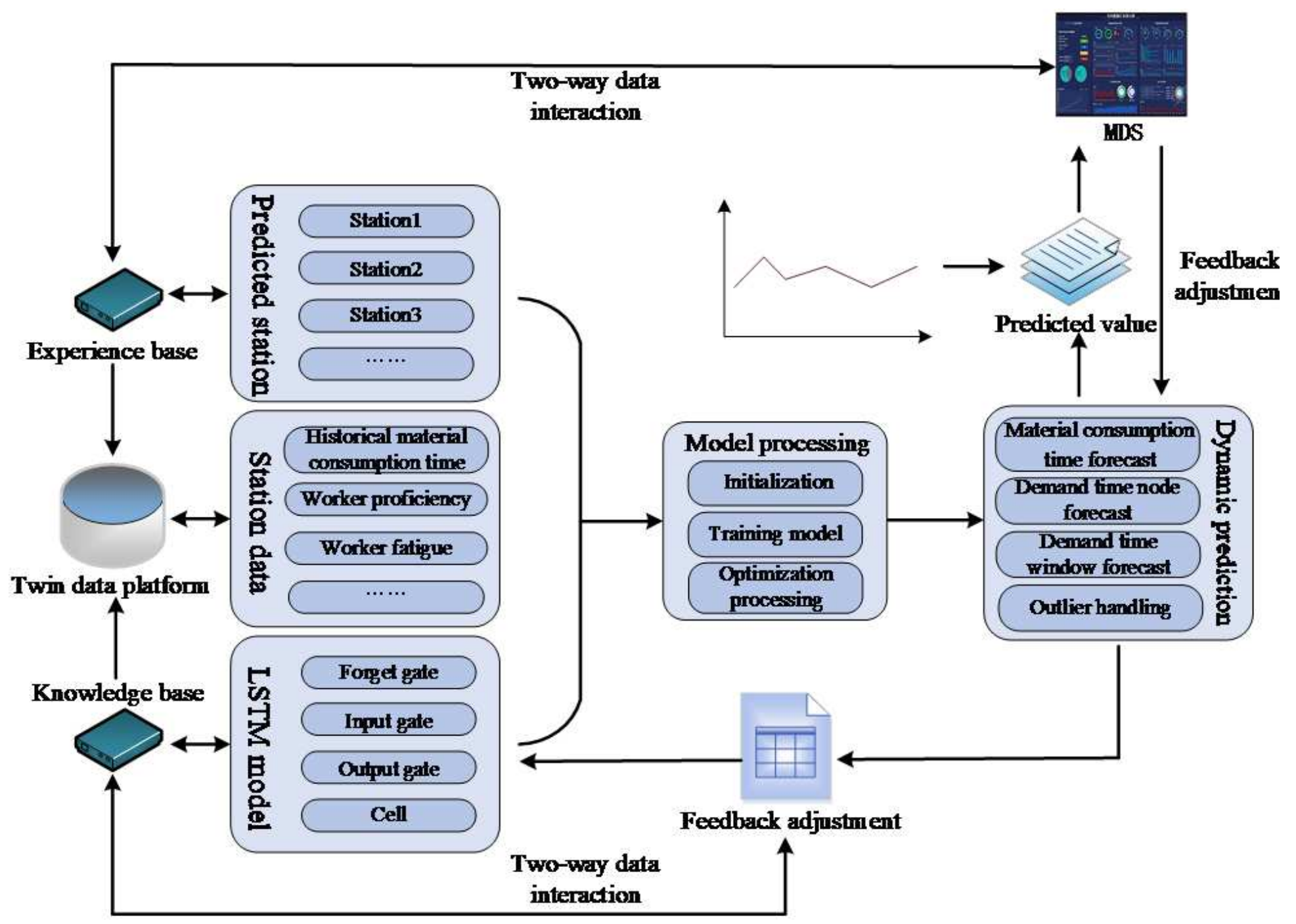

Figure 6

Dynamic forecasting operation mechanism of material demand time node 


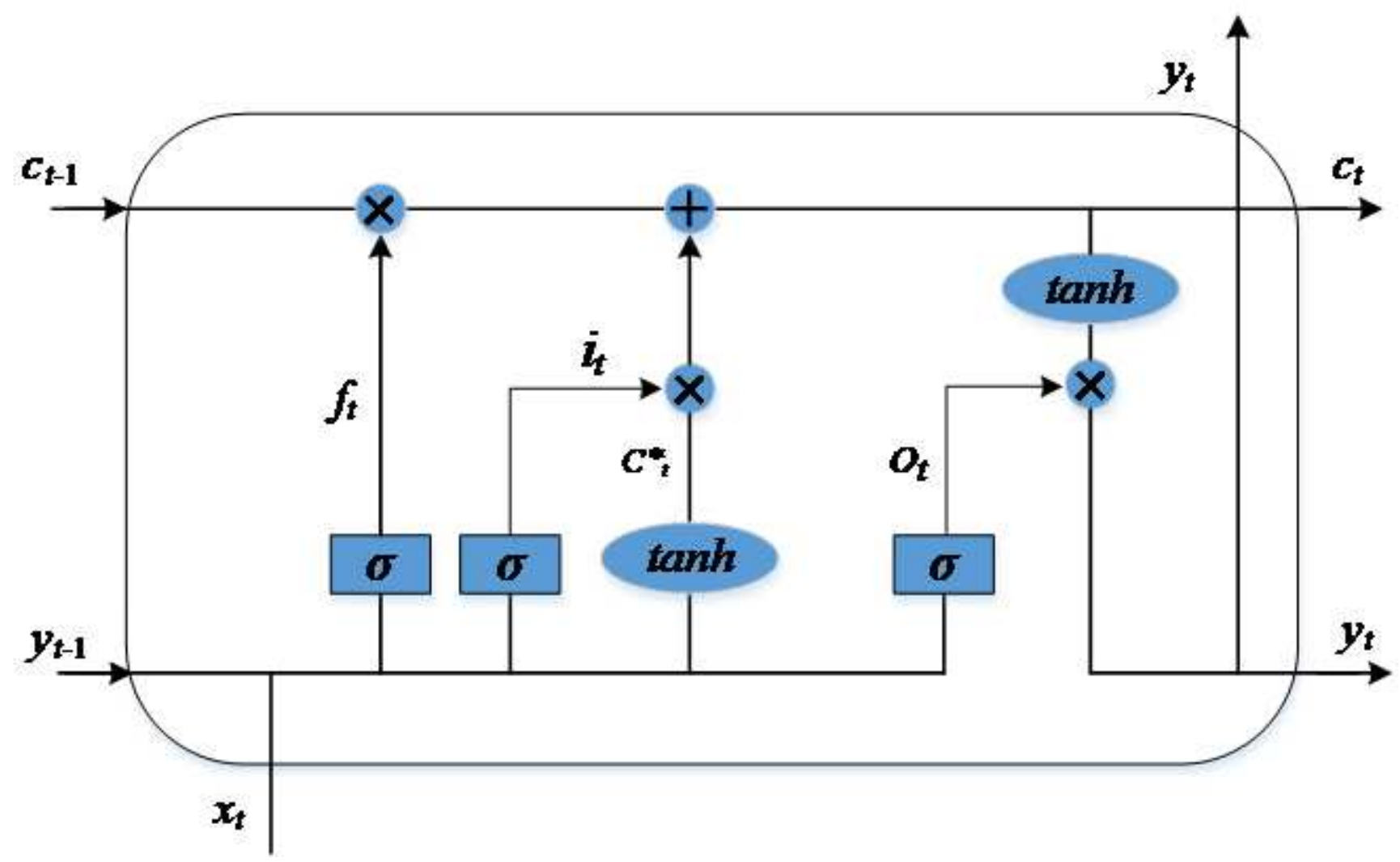

Figure 7

LSTM network model

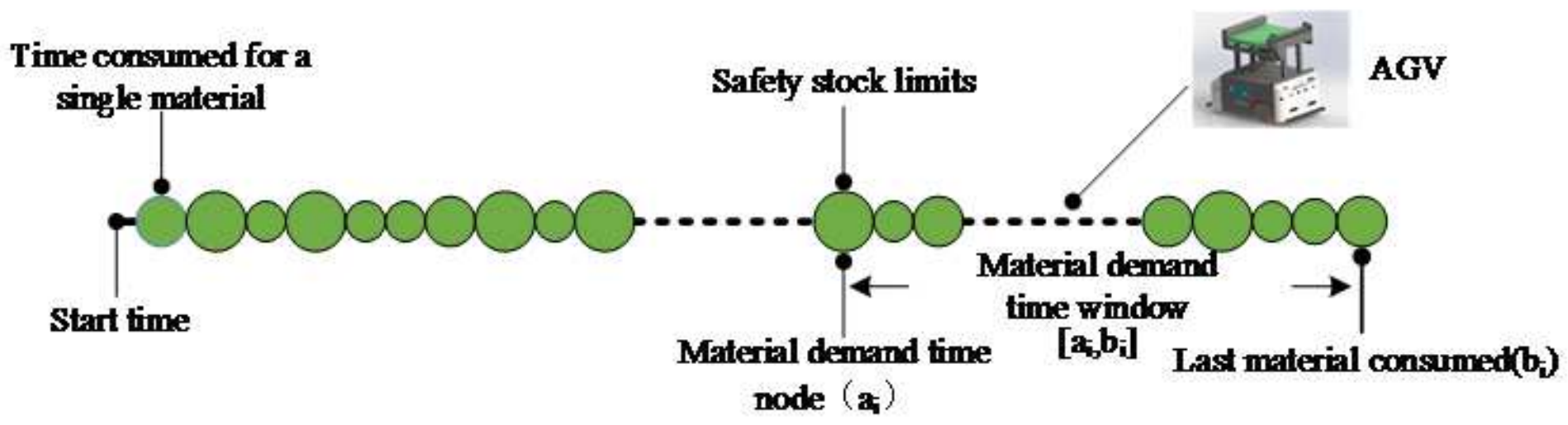

Figure 8

Time node diagram of material demand 


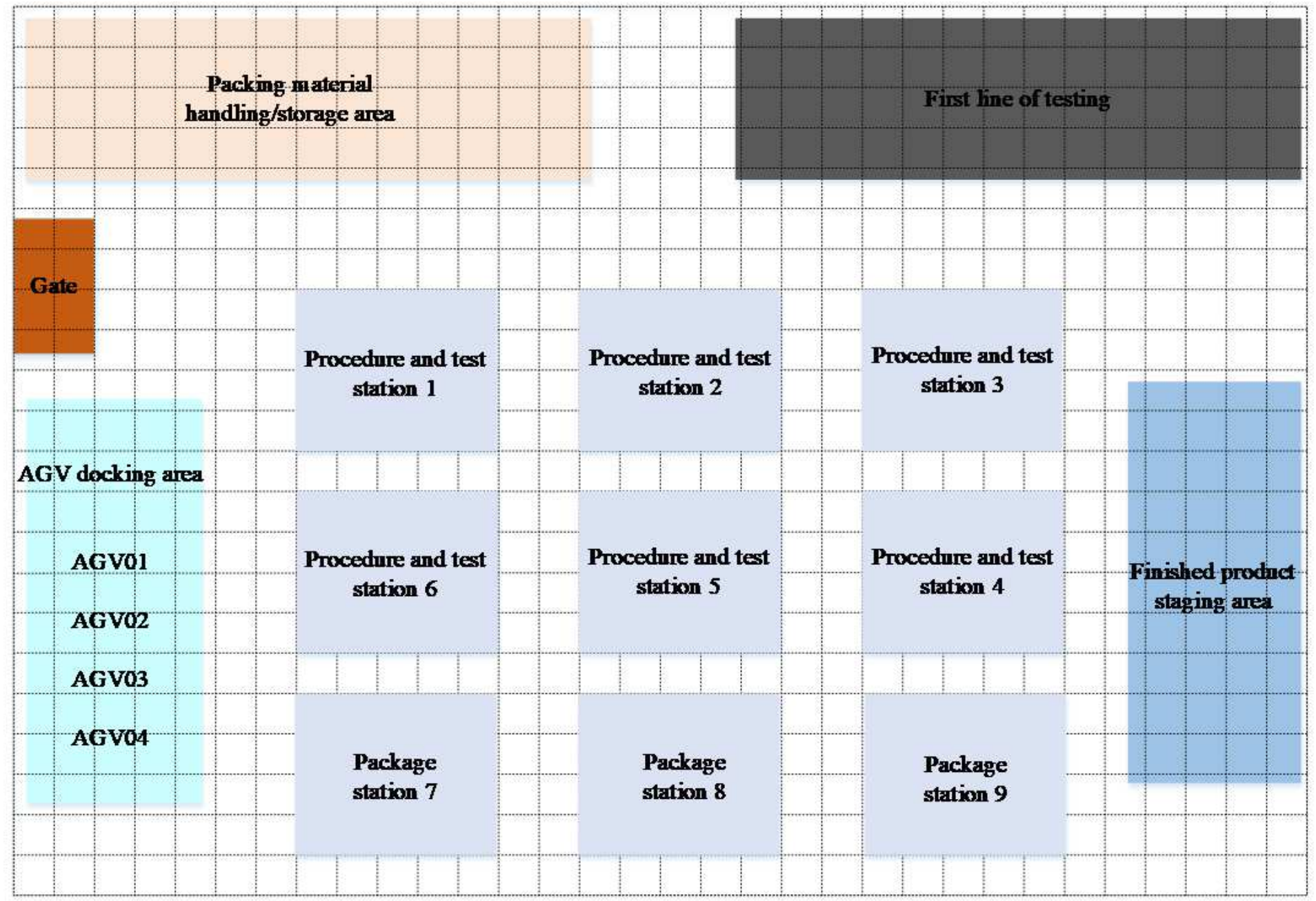

Figure 9

Workshop layout 


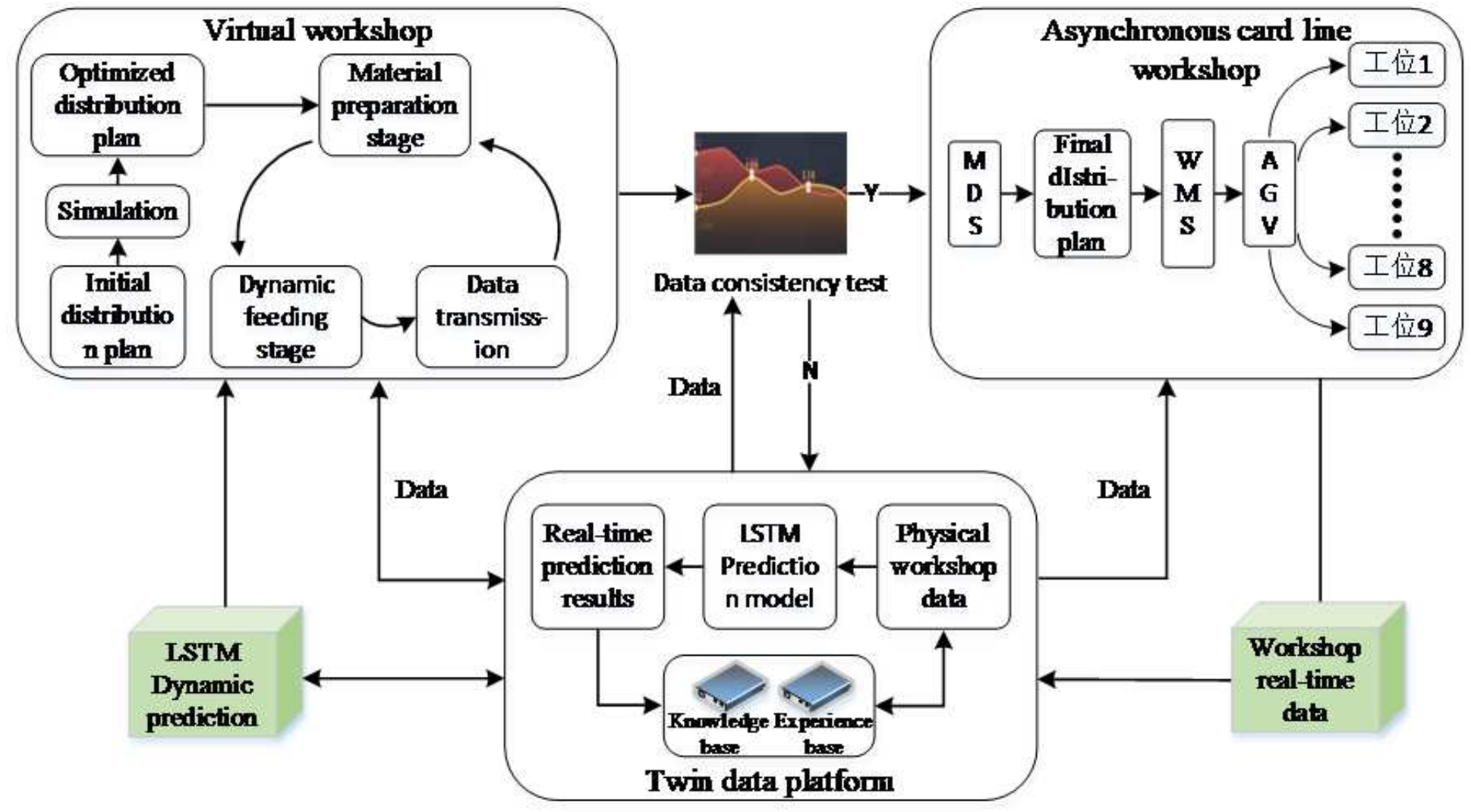

Figure 10

Material distribution operation mechanism based on digital twin 


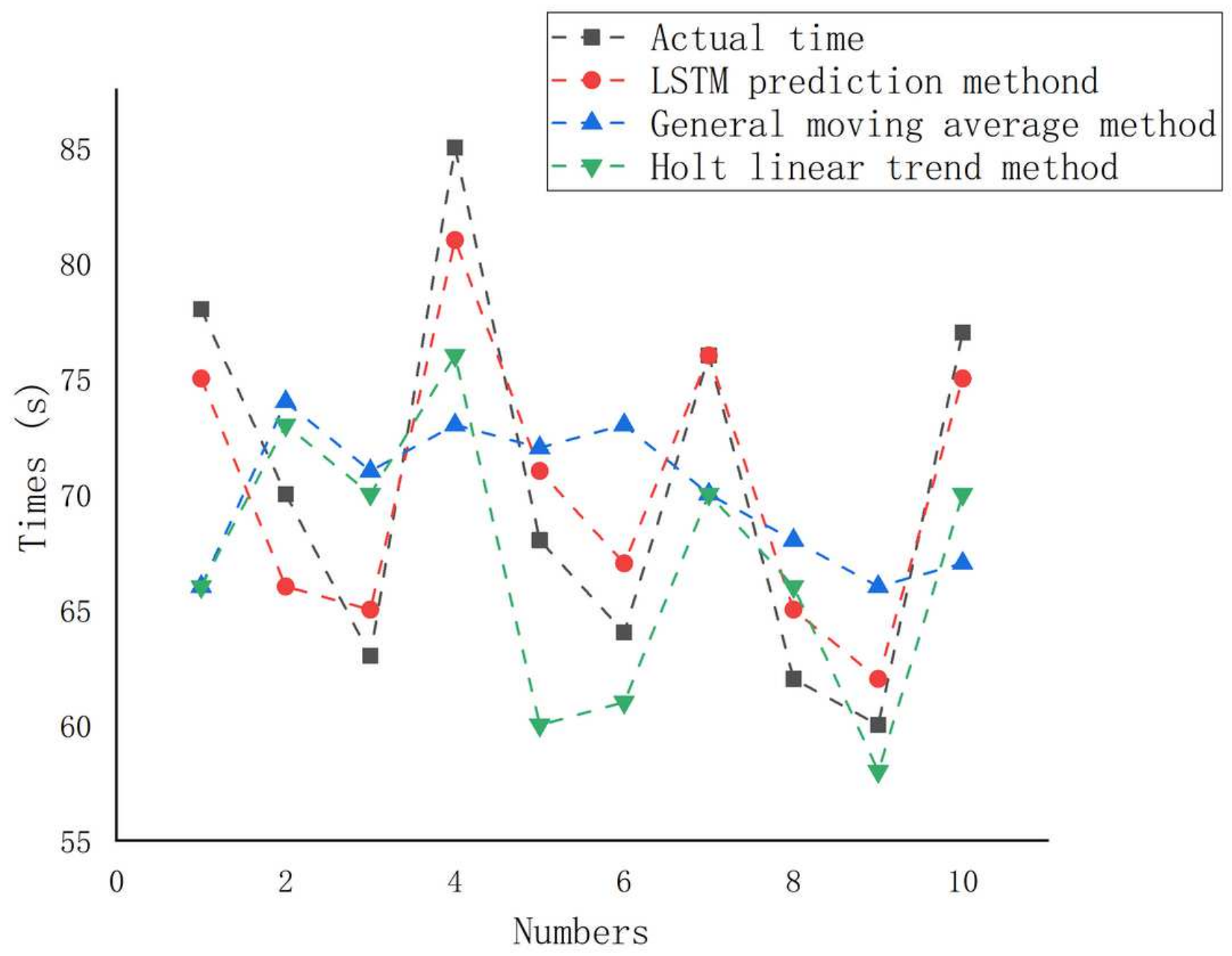

Figure 11

comparison of prediction time 


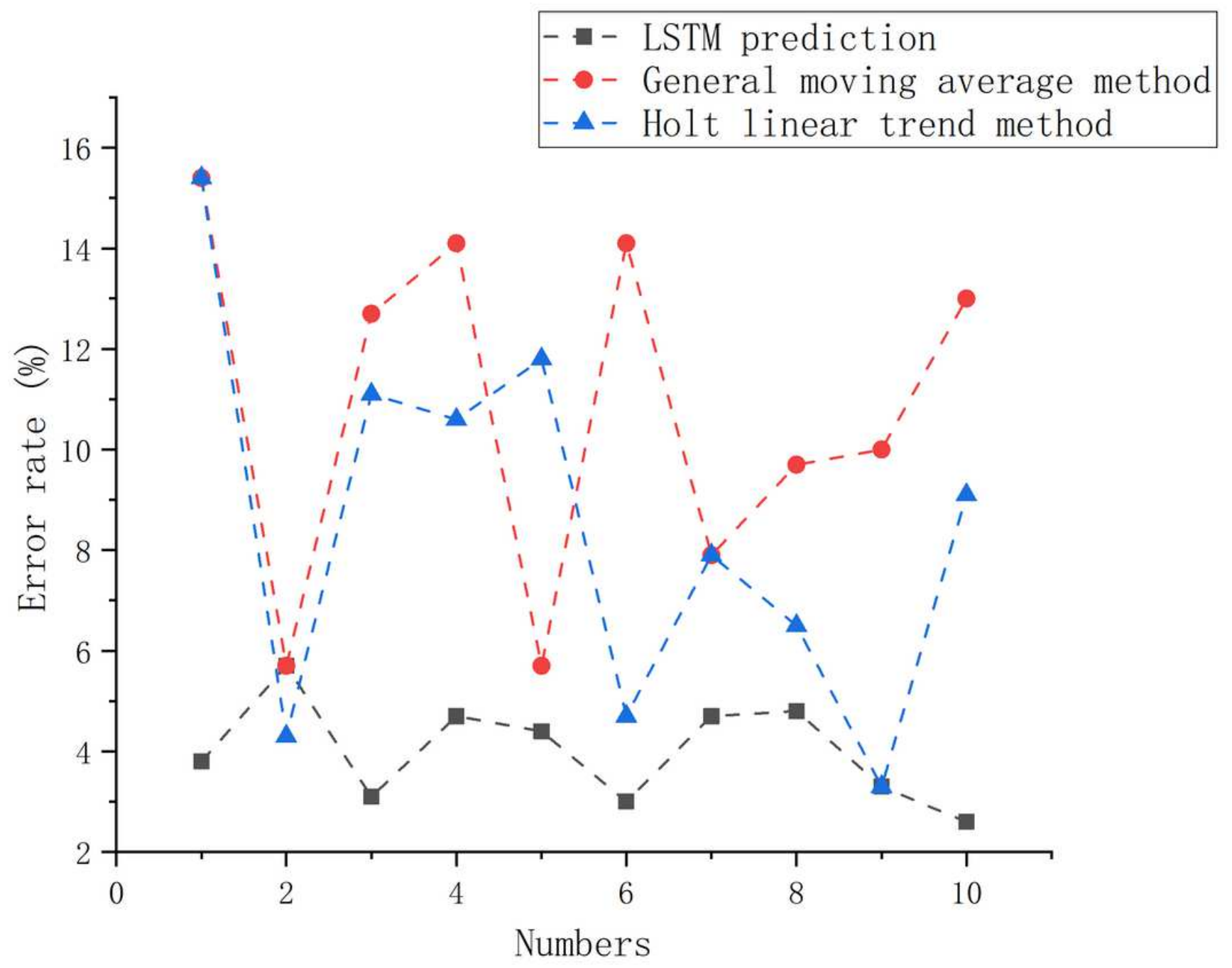

Figure 12

comparison of prediction error rate 


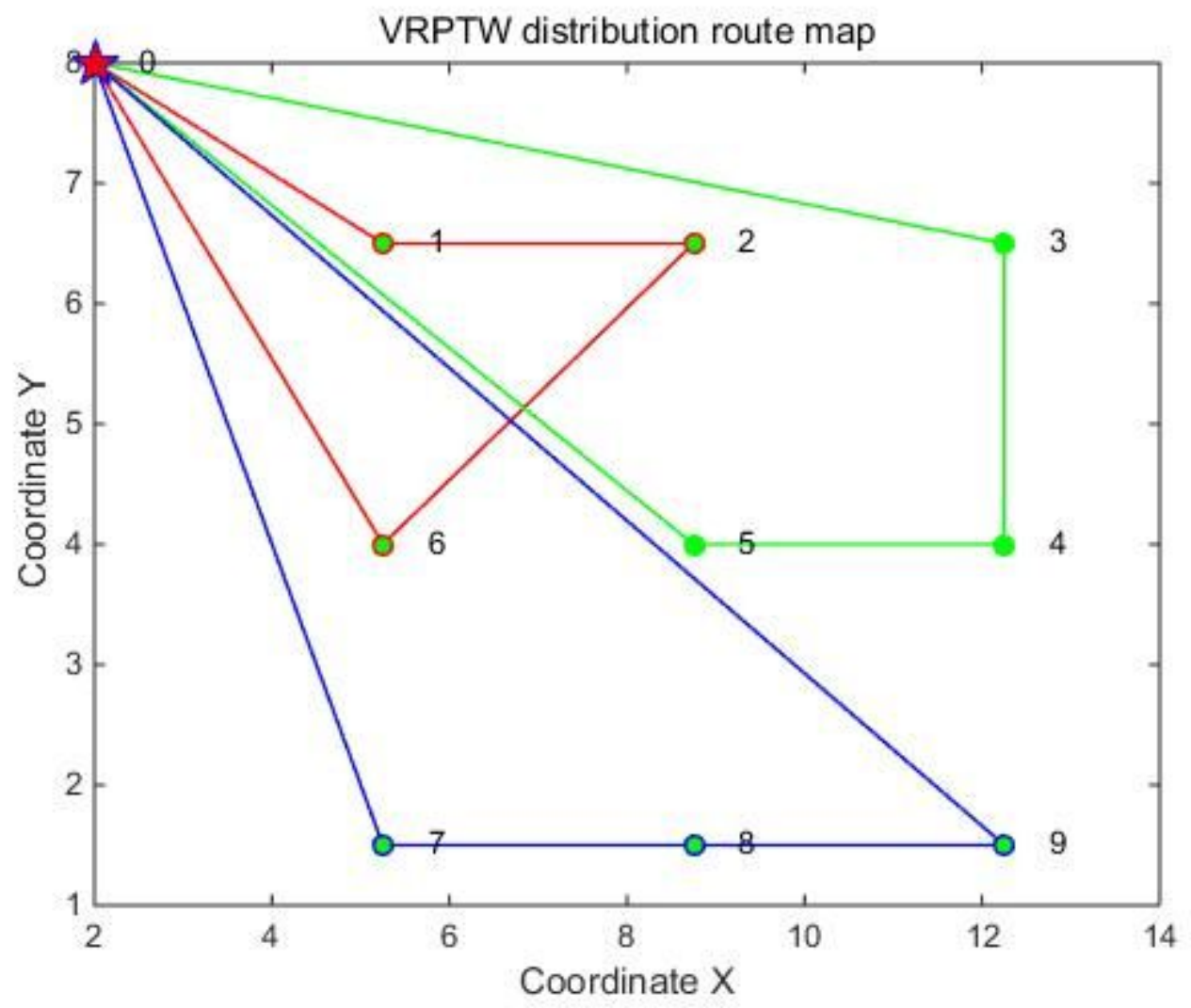

Figure 13

AGV distribution route 